\title{
BMJ Open Potential impact of encounter patient decision aids on the patient-clinician dialogue: a qualitative study on Dutch and American medical specialists' experiences
}

\author{
Ester A. Rake (1) , ${ }^{1,2}$ Dunja Dreesens (1) , ${ }^{2}$ Kristie Venhorst, ${ }^{2}$ Marjan J. Meinders, ${ }^{1}$ \\ Tessa Geltink, ${ }^{2}$ Jenny T. Wolswinkel, ${ }^{3}$ Michelle Dannenberg, ${ }^{4}$ Jan A.M. Kremer, ${ }^{1}$ \\ Glyn Elwyn (1) ,1,4 Johanna W. M. Aarts ${ }^{5}$
}

To cite: Rake EA, Dreesens D, Venhorst $\mathrm{K}$, et al. Potential impact of encounter patient decision aids on the patient-clinician dialogue: a qualitative study on Dutch and American medical specialists' experiences. BMJ Open 2022;12:e048146. doi:10.1136/ bmjopen-2020-048146

- Prepublication history and additional supplemental material for this paper are available online. To view these files, please visit the journal online (http://dx.doi.org/10.1136/ bmjopen-2020-048146).

Received 17 December 2020 Accepted 15 January 2022

Check for updates

(C) Author(s) (or their employer(s)) 2022. Re-use permitted under CC BY-NC. No commercial re-use. See rights and permissions. Published by BMJ.

For numbered affiliations see end of article.

Correspondence to

Ester A. Rake;

ester.rake@radboudumc.nl

\section{ABSTRACT}

Objectives To examine the experiences among Dutch and American clinicians on the impact of using encounter patient decision aids (ePDAs) on their clinical practice, and subsequently to formulate recommendations for sustained ePDA use in clinical practice.

Design Qualitative study using semi-structured interviews with clinicians who used 11 different ePDAs (applicable to their specialty) for 3 months after a short training. The verbatim transcribed interviews were coded with thematic analysis by six researchers via ATLAS.ti.

Setting Nine hospitals in the Netherlands and two hospitals in the USA.

Participants Twenty-five clinicians were interviewed: 16 Dutch medical specialists from four different disciplines (gynaecologists, ear-nose-throat specialists, neurologists and orthopaedic surgeon), 5 American gynaecologists and 4 American gynaecology medical trainees.

Results The interviews showed that the ePDA potentially impacted the patient-clinician dialogue in several ways. We identified six themes that illustrate this: that is, (1) communication style, for example, structuring the conversation; (2) the patient's role, for example, encouraging patients to ask more questions; (3) the clinician's role, for example, prompting clinicians to discuss more information; (4) workflow, for example, familiarity with the ePDA's content helped to integrate it into practice; (5) shared decision-making (SDM), for example, mixed experiences whether the ePDA contributed to SDM; and (6) content of the ePDA. Recommendations to possibly improve ePDA use based on the clinician's experiences: (1) add pictorial health information to the ePDA instead of text only and (2) instruct clinicians how to use the ePDA in a flexible (depending on their discipline and setting) and personalised way adapting the ePDA to the patients' needs (e.g., mark off irrelevant options).

Conclusions ePDAs contributed to the patient-clinician dialogue in several ways according to medical specialists. A flexible and personalised approach appeared appropriate to integrate the use of ePDAs into the clinician's workflow, and customise their use to individual patients' needs.

\section{Strengths and limitations of this study}

- Multiple independent coders with different backgrounds (clinicians, researchers with experience in qualitative research, biomedical scientists or a combination of these) strengthened our thematic analysis (investigator triangulation).

- Twenty-five medical specialists were interviewed based on availability of those who followed the shared decision-making training which could have influenced data saturation.

- Interviewing clinicians to ask for their experiences could have led to a restricted viewpoint which might have influenced the results.

- Interviews were conducted in two countries and showed similar results which adds to the transferability of our results

\section{INTRODUCTION}

The patient-clinician dialogue is at the core of healthcare. ${ }^{2}$ The increasing focus on the patient's role during medical encounters in the past decades has caused a shift from the paternalistic approach towards a shared decision-making (SDM) model as the preferred approach for clinical decisionmaking. ${ }^{3}$ In short, the SDM process means that the patient is informed about the options, the clinician and patient discuss possible options based on reliable information and the clinician supports the patient in exploring or eliciting his/her preferences concerning the options. Finally, the clinician and patient agree to a plan which suits the patient best. ${ }^{4}$ In the past years, a myriad of tools have been developed to support this 
process, such as patient decision aids for patients or training interventions for clinicians. ${ }^{56}$

Many studies have shown the benefits of using patient decision aids to support SDM, either in preparation of or during the clinical encounter. ${ }^{5}$ Tools to support decision-making could be classified according to a patient engagement model consisting of three categories. ${ }^{7}$ The first category consists of passive information provision by giving the patient a leaflet or showing a video. The second category adds an activation aspect. For example, the 'askthree-questions" programme prompts the patient to ask three specific questions that enhance patient involvement and information provision during consultations. ${ }^{8-10}$ The third category adds the component of collaboration between patient and clinician. Rather than providing information only, tools in this category have the specific goal to foster a genuine dialogue between the clinician and patient during the encounter. It is expected that patients and clinicians work together during an encounter to share information and views, and discuss care options. ${ }^{7}$ Such tools are often called encounter patient decision aids (ePDAs). The ePDAs differ from patient decision aids because of the intended collaboration between the clinician and patient when using the tool during the patient-clinician conversation. Contrarily, patient decision aids are 'stand-alone' tools, often used by the patient outside the consultation without a clinician involved. Examples of ePDAs are Option Grid decision aids, designed for use during the clinical encounter to facilitate the SDM process. Option Grid decision aids present frequently asked questions on available treatment options for a specific health problem in a tabular format (online supplemental file 1). These ePDAs aim to increase patient engagement, for example, by discussing alternatives and the patient's values. ${ }^{7}$ Using such encounter tools during the consultation could influence the patient-clinician's dialogue.

This influence of ePDAs on the dialogue can be viewed from three perspectives: the patient, the clinical workflow and the clinician. From the patient's perspective, a systematic review showed that using ePDAs improved patient's knowledge and awareness of treatment options, lowered decisional conflict and raised their involvement during the encounter. ${ }^{11}$ From the viewpoint of the clinical workflow, some studies focused on using ePDAs in clinical practice in general, ${ }^{1213}$ yet more insight is needed into understanding how clinicians (want to) use these ePDAs in their clinical workflow. ${ }^{14}$ From the clinician's perspective, a couple of studies already observed that clinicians did not use ePDAs to stimulate a patient-clinician dialogue but only superficially explained the ePDA. ${ }^{15} 16$ Moreover, in many clinical trials on decision aids the clinicians' perspective on using ePDAs as part of the SDM process is missing in the outcome measures. ${ }^{17}$ This missing perspective could hinder implementation of ePDAs in clinical practice. For instance, sitespecific implementation of ePDAs in America remains a challenge while many Option Grid decision aids are available. ${ }^{11}$ Simultaneously, in the Netherlands 32 ePDAs are currently disseminated via www.consultkaart.nl (from summer 2022 available via www.Thuisarts.nl) and over $€ 1500000$ (unpublished data) has been spent on development while implementation is lacking there as well. ${ }^{18}$ Although high expenditures on development of ePDAs are seen, improvements are needed to increase the use of ePDAs in clinical practice. To our best knowledge, it is not yet unravelled what the impact is for clinicians of using ePDAs during clinical encounters and in their clinical workflow. In summary, routine use of ePDAs in clinical practice remains challenging while the exact impact on the clinical encounter seems unclear from the clinician's perspective.

Therefore, our study aimed to examine the experiences among Dutch and American medical specialists on the impact of using ePDAs on their clinical practice. Based on these experiences, we attempted to formulate practical recommendations for future use of the ePDAs in clinical practice.

\section{METHODS}

We used qualitative research (semi-structured interviews) analysed by thematic analysis. ${ }^{19}$ We followed the Consolidated criteria for Reporting Qualitative research for interview studies. ${ }^{20}$

\section{Design and setting}

Experiences of medical specialists regarding the impact of the ePDA on the dialogue were collected after they used the ePDAs for 3 months. In New England (USA), Option Grid decision aids were used while in the Netherlands their Dutch equivalents called "Consultkaarten" were used. Both will be indicated as ePDAs in this study. The American part of the study was performed at the Dartmouth-Hitchcock Medical Center (DHMC) in Lebanon, New Hampshire, and the affiliated community group practice, Putnam clinic in Bennington, Vermont. In the Netherlands, the study was performed in nine hospitals (Amsterdam UMC location AMC, Radboud university medical center, Máxima MC, Maastricht University Medical Center+, Kempenhaeghe, Haaglanden Medical Centre, ViaSana, Canisius Wilhelmina Hospital and, Dijklander Hospital).

\section{Patient and public involvement}

For this study, neither patients nor public were involved in our study design to interview clinicians, as we focused on clinician's experiences. However, patients were involved in the former development process of the ePDAs that we used. In the USA, Option Grid decision aids were developed and pilot-tested with patients. In the Netherlands, development and pilot-testing was done in collaboration with the Netherlands Patients Federation. Subsequently, these ePDAs are supposedly simple to use, the content is evidence-based and developed without conflicts of interests. 


\section{ePDA training}

Introduction of the ePDAs has been similar in both countries, led by the same research group (GE and JWMA). During the preparation phase, clinicians received a group-based training on how to use the ePDA during the encounter and the relation of the ePDA with SDM. Details on this training are described elsewhere. ${ }^{21}$ One part of the training differed slightly between countries: in the Netherlands, clinicians received written instructions; in New England, role playing was part of the group-based training. KV visited the participating Dutch hospitals to give the training. JWMA provided the training in New England. Medical specialists were asked to use the ePDA at least three times during the 3-month study period. Online supplemental file 2 shows the ePDAs used in this study. Subsequently, after 3 months of usage, semistructured in-depth face-to-face interviews took place.

\section{Participants}

In New England, gynaecologists and medical trainees in two clinics were recruited for participation. In the Netherlands, different medical specialists from the nine hospitals were asked to participate. All clinicians who followed the SDM training for ePDAs in New England and the Netherlands were invited by email to participate in an interview. Those who did not reply after 2 weeks received a reminder. Moreover, clinicians who did not use the ePDA during our study were also explicitly asked to participate in the interviews in order to gain knowledge about nonusage, and to generate results for improved use.

\section{Data collection}

The research team consisted of several senior researchers with much experience in the field of qualitative research (GE, JWMA, DD, MJM and JAK). They guided and educated the junior researchers (KV, TG, MD, JTW and EAR) during data collection and analysis; in addition, two junior researchers (KV and EAR) followed a course on conducting qualitative research. We developed the interview guide, initially for the US evaluation, based on literature, experiences from the research team and previous experiences from implementation projects, for example, ePDA studies on circumcision, knee osteoarthritis and breast cancer. ${ }^{12} 1322$ This guide was translated into Dutch and adapted to the local context for the Dutch part of the study. Online supplemental file 3 shows the American interview guide. The adapted and translated Dutch guide is available on request. Both guides were tested (and adapted when necessary) within the research team before the interviews took place. Interviews have been conducted by native speakers (in the Netherlands, TG did the interviews; in the USA, an independent research assistant conducted the interviews). The interviewers (both women) were neutral, that is, they were not involved in any step related to the development of the ePDAs or in the preparation phase of this study. Consequently, the interviewers were less biased by preconceived ideas of how to use the ePDAs in clinical practice.

\section{Analysis}

Thematic coding was chosen because it provides a flexible strategy for analysing rich, detailed and complex qualitative data. Themes were identified at a semantic level through the iterative analysis process described below, recognising topics directly communicated by the clinicians. All interviews were audio-recorded with consent of all participants. Verbal consent was recorded for the American interviews; in the Netherlands the clinicians gave written consent. Interviews were transcribed verbatim and identifiers were removed from the transcripts. To ensure data accuracy, all participants received their transcript and agreed about the content (member checking). The researchers who performed the analysis consisted of clinicians (gynaecologists), researchers with expertise in qualitative research and biomedical sciences, advisors of knowledge tools or a combination of these professions. The diverse background and having both clinicians and researchers included in our team gave us the possibility to conduct the analysis from several perspectives. We followed the six steps of thematic analysis, starting with familiarising ourselves with the data. First, all researchers involved in the analysis process listened to the audio-recordings, read and re-read the Dutch transcripts (KV, TG, JWMA and EAR), and the American transcripts (JTW, MD, JWMA and EAR). Subsequently, the second step consisted of generating initial codes followed by searching for initial themes (step 3). Two researchers (JTW and MD) independently coded the American transcripts and two researchers (KV and TG) independently coded the Dutch transcripts using ATLAS.ti. All codes from both study sites were discussed with a third researcher (JWMA) during regular face-toface meetings and conference calls. Disagreements were solved by discussing codes with a third coder (JWMA), for both the Dutch and American interviews. All codes were checked by EAR before integrating both code sets. The underlying quotes and texts were consulted after reading all interviews. The code sets were integrated by discussing each code (JWMA and EAR), rearranging and merging codes if necessary and until consensus was reached. This led to a final codebook (EAR) from which we derived the initial themes. The coding process involved many researchers (investigator triangulation) with discrepancies discussed until consensus was reached, which was done to minimise bias during data analysis.

Step 4, reviewing themes, was done through an iterative process performed by JWMA and EAR. The initial themes were reviewed and thoroughly compared with the coded extracts, categories and our research question. This process led to the defining and naming of the final themes (step 5). Finally, we produced our report (step 6 of thematic analysis), where we selected compelling extracts to support the identified codes and themes. 


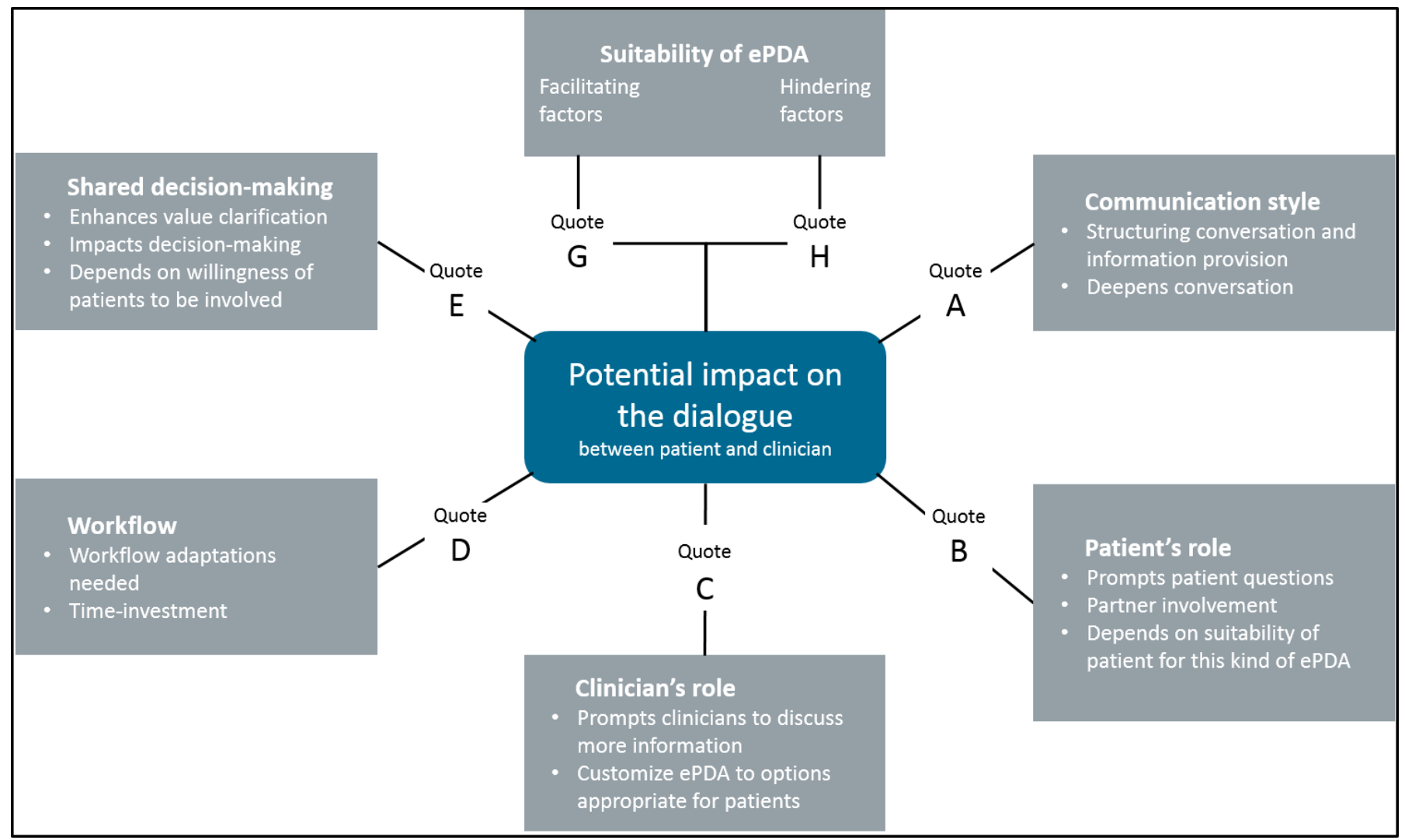

Figure 1 Overview of the six themes (grey blocks) that have potential impact on the dialogue using an ePDA (blue block). The lines refer to the contributions of the themes to the concept we studied, exemplified by quotes (see results). ePDA, encounter patient decision aid.

\section{RESULTS}

\section{General results}

In total, 25 medical specialists participated in the interviews. Sixteen were Dutch medical specialists (ie, seven gynaecologists, five neurologists, one orthopaedic surgeon and three ear-nose-throat specialists) who used one or more ePDAs related to their specialty. In New England, nine American gynaecologists used one or two ePDAs. Seven of them worked at DHMC, two worked at the affiliated hospital in Bennington. Of the clinicians in DHMC, four were gynaecology medical trainees. Due to time constraints, one interview took place via telephone instead of face-to-face. Interviews lasted an average of 30 min (range 16-49 $\mathrm{min}$ ).

\section{Interview results}

The interviews were analysed using thematic analysis. This analysis was focused around the concept of the potential impact of the ePDA on the patient-clinician dialogue. Several aspects were identified that influenced the dialogue where an ePDA was used according to the medical specialists. Further scrutiny of the transcripts showed six themes that influenced the extent to which the ePDA impacted the patient-clinician dialogue. These six themes included (1) communication style; (2) the patient's role; (3) the clinician's role; (4) workflow; (5) shared decision-making; and (6) content of the ePDA. The contribution of each theme to the main concept is depicted in figure 1, where letters in the arrows refer to the quotes; NE quotes are quotes from clinicians in New England; NL quotes are quotes from clinicians in the Netherlands. The quotes give a deeper insight into the contribution of each theme to the concept we were interested in.

The six themes each contributed to the concept of the potential impact of ePDAs on the patient-clinician dialogue. For example, the ePDA prompted the patient to ask more questions. In this way, the theme 'patient' impacted on the patient-clinician dialogue. Alternatively, the theme 'content' impacted the dialogue in such a way that clinicians reported a clear overview of information to discuss due to the content, but the amount of information could overwhelm the patient. Both aspects of the theme 'content' impacted on the dialogue as a consequence of using the ePDA.

Resulting from the medical specialists' experiences and the themes, we identified practice recommendations for future use of ePDAs in clinical practice. The themes and identified practice recommendations will be elaborated on in the following sections. Online supplemental file 4 shows the underlying codes.

\section{Communication style}

Two codes contributed to the potential impact of the ePDA on the clinicians' communication style. First, clinicians reported that the ePDA changed the dynamic of 
the conversation and deepened it. Second, clinicians perceived the ePDA helpful in structuring the dialogue and providing information. It made them feel confident having covered all viable options.

A. And, but it was nice and it gave a structure to the discussion and it, you know, reminded you of all of the things to talk about. (NE4)

\section{Patient's role and suitability}

The overall experience of patients, as perceived by clinicians, was positive. On the contrary, two clinicians from both settings commented that patients did not seem to listen as well anymore while focusing on the ePDA.

Many positive aspects were mentioned by the clinicians regarding perceived patients' experiences. According to them, the ePDA structured information for patients and patients expressed their thoughts and concerns about the applicable options. Patients really felt encouraged to ask more questions. Some Dutch clinicians reported that the therapy compliance of patients improved because of the ePDA. A few clinicians noted that partners of patients were more involved in the conversation due to the ePDA. Furthermore, some clinicians reported that patients had more realistic expectations of the chosen treatment.

B. Especially when you're talking about the effectiveness of a treatment or side effects of a treatment or possible complications of a treatment. And if anything of those things happens that they feel like that they knew that there could be side effects or complications and that they themselves made a choice, to do something anyway, and that it wasn't a choice that was forced on them. (NE8)

An important negative aspect involved the perceived suitability of the patient for ePDA use. Clinicians did not use the ePDA with patients they deemed not suitable, such as illiterate patients, patients with intellectual disabilities or patients with a language barrier.

B. The women who are more autonomous, they liked the ePDA because they can make their own plan beforehand. Others thought like: 'yeah I don't know, what's your advice doctor?'. For some women it worked well, others just really appreciated my opinion. (NL10)

It was difficult to use the ePDA with patients who had strong prior beliefs on what they want. For this group, clinicians perceived the added value of the ePDA as low, since the patients were not receptive to hear other possible options. The general feeling of the clinicians was that it depends on the type of patient which ones would benefit from the tool. They reported that the most suitable patients were patients who are in doubt or do not have a clear opinion towards any option.
Clinician's role

Clinicians reported that the ePDA supported them in educating the patient, that they felt more confident in having covered all options and that they were able to focus on the patient's preferred treatment after discussing the options. Using the ePDA encouraged them to discuss more information during the encounter. Clinicians noted that they customised the ePDA to options appropriate for the specific patient sitting across the table. They marked off options they deemed inappropriate for that specific patient.

C. I would write on it, and mark on it and kind of go over it what we said with the top two or three and you know, options, and I would hand it to them. (NE9)

Numerous clinicians experienced that their patients liked the tool and reacted positively. Some clinicians added that their own bias could have influenced this, since they chose with whom to use the tool.

C. You know, you ask people of whom you think: they can handle that.... I can imagine that you do not give the ePDA to everybody. (NL11)

Only Dutch clinicians explicitly reported aspects regarding the patient-clinician relationship. Three clinicians emphasised that the ePDA contributed to a better connection and improvement of the patient-clinician relationship, of whom two specifically reported that the tool enhanced mutual respect and equality between patient and clinician.

\section{Adapting workflow}

Clinicians experienced difficulties fitting the ePDA into the existing workflow. For example, neurologists found it hard to find the right timing to use the ePDA, since giving a patient an epilepsy diagnosis is already difficult. They recognised that patients were overwhelmed by the diagnosis and were not capable to use the ePDA as immediate treatment was necessary.

Many clinicians indicated that after using the ePDA a couple of times, they found it easier to use. Familiarity with the mode of use helped them to apply the ePDA in clinical practice. This also decreased their feeling of needing extra time.

I did, think that I definitely got faster at it the more I did it, there is kind of a curve that you know, developing your spiel for how you talk about $\mathrm{AB}$, around this grid [ePDA]. (NE6)

The most-heard challenge was to find enough time to use the ePDA during an encounter. The interviewees had several strategies to integrate the ePDA in the existing workflow. A recurring strategy was to first go through the ePDA with the patient, then give time to read the ePDA and thereupon discuss any patient's questions (as was explained during the training). Others first counselled a patient, then gave the ePDA to read while in the waiting room, in the meantime seeing another patient. 
Subsequently, they recalled the first patient to discuss options and questions. Some clinicians thought that using the ePDA could eventually save time, because the decision made would fit better with the patient's preferences.

D. In the beginning I thought I should apply it [using the ePDA] as was shown to us in the training. Then I thought, no I should do it the way it works for me and integrate that. So it was a bit of a search for me. ... I think it is important to repeat that clinicians should use it in their own way, that a kind of freedom is very important. (NL1)

Some Dutch clinicians expressed that being in salaried employment influences the use of the ePDA in clinical practice compared with non-salaried employment. They said that they have more time per patient in academic hospitals compared with clinicians working in general hospitals, because the former are not dependent on patient volume for their income. They thought being in non-salaried employment could negatively influence the use of the ePDA for their peers working in general hospitals.

Finally, clinicians reported that they had a hard time to cope with options in the ePDA that were inappropriate for the patient. Not all options are applicable to each patient and they had to find out how to deal with that.

\section{SDM process}

Clinicians agreed that the ePDA seemed to help patients in the process of SDM, that is, involving them in which options are available, discussing (dis)advantages per option and having access to reliable information, discussing values and preferences and making a final decision.

There was no agreement about whether the ePDA contributed to this final step of making the decision itself. Most clinicians said that the ePDA helped patients to make decisions, that is, that patients were able to make a fully informed choice. Some thought that it did not influence the final decision being made, as they explained that patients did not choose differently or that they were not sure whether patients would have acted differently without the ePDA. Some clinicians reported very specific situations in which the patient made a different decision because of the ePDA.

E. It surprised me, because of the ePDA, people made choices that I would not have thought about beforehand. One person really valued not losing hair. Another patient had such a specific thing as well. So it was surprising. I think this is the big advantage for people, to have insight in the options and make decisions well-informed. That they are aware of side effects. (NL15)

Several clinicians from both countries mentioned that the ePDA enhanced value clarification in the decisionmaking process of the patient, which is part of the SDM process. However, one clinician disagreed on this and expressed that value clarification was achieved because of the clinician's conversation techniques rather than the ePDA itself.

Other topics raised related to SDM was that the ePDA could also support patients with future choices or when they have to reconsider options. Finally, clinicians commented that not all patients want to use the ePDA or want to be involved in decision-making.

\section{Suitability and provided format of the ePDA}

The content and format of the ePDA both facilitated (quotes G) and hindered (quotes $\mathrm{H}$ ) the conversation. Clinicians were generally positive about the ePDA. For example, they shared that if you provide information on a leaflet, patients will remember the content much better compared with information only told to them during an encounter. Their experience was that the patient liked to have the tool as a reference.

F. Because it is shown that half the time they don't hear what we are saying, to notice that, absolutely, and that's the point of being able to go home with written material and internet material also that they can review it at home when they are not so nervous. (NE7)

Furthermore, clinicians mentioned that the information on the ePDA is more neutral compared with when clinicians tell it.

F. Actually, I thought we already do that [give neutral information], but that's not the case. Thus, by using the ePDA it occurred to me that it helps patients to use the ePDA a lot. (NL13)

Other facilitating factors were clear, complete and reliable information which gives insight in the available options. A Dutch clinician explained the difference between the ePDA and local patient information as follows: the ePDA provides information on all treatment options. A local patient leaflet discusses one option only, in the specific context of the local hospital organisation.

Hindering factors were mainly due to the amount of information. Many clinicians were concerned that the amount of information might overwhelm patients. Furthermore, the ePDA contained a lot of information, which felt as too much to cover within one encounter. However, clinicians reported conflicting experiences regarding the amount of information.

G. It takes some time to explain that there are no exhaustive answers on the ePDA. Sometimes you receive reactions like: 'yeah but it is not differentiated enough because for epileptically surgery, a lot of percentages are relevant. It is not for sure that the chance is large'. While at the same time, the same colleague tells another colleague on the same day: 'yeah, but it contains a lot of information. You should omit the details, because it is too much'. (NL8) 
Table 1 Identified improvements for sustained use of the ePDA in clinical practice

\begin{tabular}{ll}
\hline Topic & Codes \\
\hline The ePDA itself & Make ePDA customisable for provider. \\
& Potentially make into flow chart or \\
& application. \\
& ePDA is too busy. \\
& Add pictures. \\
& More ePDAs for different situations would \\
& enhance applicability.
\end{tabular}

Introduction and Best way to use ePDA is unclear. implementation in practice

\section{Important to give clinicians a good briefing about ePDA.}

Remind each other to use ePDA in clinical practice.

Hearing positive experiences of colleagues.

Added value of ePDA should be proven.

Time investment to use ePDA in clinical practice should be studied.

Implementation should start by educating medical trainees.

Many shared decision-making initiatives, overview is lacking.

Improve publicity of ePDA towards patients and general practitioners.

$\begin{array}{ll}\begin{array}{l}\text { How the ePDA } \\ \text { is used during } \\ \text { consultation }\end{array} & \begin{array}{l}\text { Integration of ePDA into database or } \\ \text { electronic patient record. }\end{array} \\ & \text { Discuss ePDA with patient. } \\ & \begin{array}{l}\text { Go through it with patient, then give time } \\ \text { for patient to read by themselves and } \\ \text { then discuss questions. } \\ \text { Patients took ePDA home after } \\ \text { discussing, as reference. }\end{array}\end{array}$

ePDA, encounter patient decision aid.

Another reported negative factor was that the ePDA did not always fit within the local context. Sometimes an option in the ePDA is not available in the clinic where the consultation takes place. This was particularly difficult for medical trainees, who depend on their medical specialists who finished training to confirm the treatment approach.

G. A lot of the attendings [medical specialist who finished training, red.] won't do ablations and so that very ... it's just one of the options on the grid [ePDA] but it may not be an option. Or at least with that attending that day or that time. Or they may want further evaluation or they need to be referred to somebody else to happen. So it can be a very tricky situation that you have worked yourself into. To say like here are your options, this is really not a sort of an option. So I had one patient in particular that was angry that she felt like it was her right to get that and we didn't schedule for her that day [in our clinic].' (NE2)

\section{Recommendations for future use in clinical practice}

Resulting from the clinicians' experiences and factors that might positively or negatively influence the impact on the dialogue, several recommendations could be formulated to enhance use of the ePDAs in clinical practice. We identified three main topics for recommendations (table 1).

The first topic consists of ideas on how to improve the ePDA itself. To improve understanding of the information, it was suggested to add pictures or adapt the ePDA into a flow chart or application.

It needs some visual, it needs some pictures. There are too many words. So in terms of actually talking to a patient about it, I lined up drawing pictures on a separate piece of paper because the patient doesn't know what her uterus looks like. She doesn't know what the options look like. (NE3)

Furthermore, Dutch clinicians proposed ePDAs in different languages for those patients that do not sufficiently understand the Dutch language.

The second topic shows ideas on how to implement the use of the ePDAs in clinical practice. Some clinicians said that the best way to use the ePDA was unclear to them. Furthermore, encouraging your colleagues to use the ePDA was often-mentioned to improve the implementation of the ePDA in clinical practice.

But what's important: if I show right now, that the patients coming to me are so much happier, better, than for sure my colleagues will do so [use the ePDA]. (NL4)

The third topic related to ideas and struggles on how to use the ePDA during the consultation. Several clinicians suggested giving the ePDA to the patient before the encounter, either while in the waiting room or mailing it to them before the appointment (when the reason/ diagnosis is clear). During the encounter, the clinician and patient can discuss the options. In their opinion, this could improve the conversation and prepare the patient for the encounter.

I think it would be really helpful if they have a chance to take a look at it before their visit $[\ldots]$ if they are a little bit familiar with the option grid when you're going through with them, then it makes it easier to kind of guide the discussion, I would say. (NE1)

Other clinicians thought that some patients will be confused to see options that eventually turn out unsuitable for them.

I would hesitate to do that [send ePDA home before encounter] because for some patients like, you know, certain types of management might not be as like us, you know, they should know all their options but 
you know typically for instance like if someone has fibroids and it's in the cavity then you can't place a mirena IUD, they are gonna see maybe mirena IUD as an option for these certain situations and not understand what their pathology is and kind of be like 'why can't I have that', you trying to explain to them, so I feel like in that way it might be contra productive. (NE5)

\section{DISCUSSION}

Our qualitative interview study among Dutch and American medical specialists identified six themes that contributed to the potential impact of ePDAs on the patient-clinician dialogue. In short, the ePDA influenced the communication style (theme 1), prompted both patients and clinicians to discuss information (themes 2 and 3), asked for a change in workflow and flexible use (theme 4), influenced the SDM process (theme 4) and steered the dialogue because of the positive and negative factors of its content (theme 6). Based on the clinicians' experiences, this study also identified topics for increased and sustained use of ePDAs in the future.

Overall, our results hold two relevant lessons for practice: (1) the ePDA could contribute to the patient-clinician dialogue and (2) customised use of the ePDA and a flexible workflow could improve the uptake into routine practice. The first lesson is supported by literature about Option Grid with the most important difference that those studies were performed in one centre, with only one ePDA or in just one medical specialty. For instance, a study evaluating an Option Grid for osteoarthritis of the knee showed that the extent of observed SDM during encounters raised, measured by the OPTION score. ${ }^{12}$ Another qualitative monocentric study on using an Option Grid for neonatal circumcision reported that the tool facilitated discussion between clinicians and patients. ${ }^{13}$ Clinicians reported that the Option Grid enabled collaboration, preference clarification and information provision. These are all important aspects of SDM.

The results consolidate that the dialogue is facilitated using ePDAs by showing similar results for different ePDAs, across different specialties in two different countries. Although clinicians in our study were not unanimous whether the ePDA enhanced SDM, they agreed that it contributed particularly to clarification of the patient's values in the decision-making process. This specification provides more insight on how the ePDA might facilitate the discussion from a clinician's perspective.

The second lesson of our study across several disciplines in two countries suggests that the customised use and flexible workflow are important elements to reach sustained ePDA use in routine settings. For some clinicians, it worked well to let the patient read the ePDA while in the waiting room, others did some computer work while the patient read the ePDA during the encounter. This depends on the specific care-pathway and organisation in the department. In order to overcome the barrier of integrating the ePDA in the workflow, site-specific features are essential to take into account. ${ }^{2324}$ For example, organising access to the ePDA for patients before consultation deserves attention. ${ }^{25}$ Some clinicians mentioned making the ePDA accessible before the consultation for patients with a clear diagnosis. This might be an important strategy to improve implementation because the patients can bring up the ePDA themselves. Another advantage of giving patients access to the ePDA before their consultation could be that they have time to synthesise their preferences, because constructing preferences usually takes some time. The customised use of the ePDA is also related to individualising the ePDA to the patients' needs during the encounter. Not all clinicians were aware that they could adapt the ePDA according to the patients' needs, for example, by marking off irrelevant options.

\section{Interpretation of results}

Our results are related to three important aspects of the patient-clinician dialogue, namely SDM, patient-centred communication and the ePDAs content. Following the principles of SDM, ePDAs could improve the quality of the dialogue between clinician and patient. Based on our results, ePDAs seem to facilitate some important components of the SDM process such as structuring the conversation and clarifying values. ePDAs were originally developed to facilitate all steps of the SDM process (presenting options, discussing the alternatives, deciding together).${ }^{146}$ However, some of these steps, such as preference elicitation, are not mentioned at all by clinicians when using these ePDAs in practice. ${ }^{1426}$ More research is needed on how this specific step of preference elicitation might be influenced by the ePDA and how to mitigate such effects.

Regarding patient-centred communication aspects, our study suggests that clinicians experienced a valuable dialogue with the patient using the ePDA. They experienced more mutual respect and a deepened conversation which contributed to the dialogue's quality. Especially, individualising the dialogue appeared to be facilitated by the ePDA. Given the nature of the ePDA, it is possible to select topics for the conversation relevant to the specific patient. The ePDAs could help to personalise the encounter and structure the conversation according to the patient's needs. Some clinicians in our study thought they needed to explain all options of the ePDA; however customising the ePDA to the patient's situation, for example, by marking off irrelevant options, might save time while still complying with the intended use of the ePDA. ${ }^{21}$ There is some room for improvement such as explaining clearly to clinicians how they can use the ePDAs in a flexible personalised way. This is in line with the acknowledgement of clinicians that there exists a learning curve to be able to use the ePDA in their conversation with patients, which is supported by other studies on Option Grid. ${ }^{13} 27$ 
Regarding the ePDA's content, clinicians mentioned that the ePDAs contained much text while pictures could further clarify the content, especially for illiterate patients. This is consistent with other studies, such as the development of a well-received 'picture Option Grid' for patients with breast cancer. ${ }^{28} \mathrm{~A}$ systematic review on the effect of pictures in health information suggested that especially for lower health literacy populations, pictorial health information increased knowledge and understanding. ${ }^{29}$ In the Netherlands, one out of seven adults has low literacy skills ${ }^{30}$ while in the USA this rate is even higher. ${ }^{31}$ Adding illustrations is an important step in making ePDAs accessible for all kinds of patients with the aim to foster the patient-clinician dialogue. When updating the content of the ePDAs it should be taken into account that pictures or infographics could improve the usability of the ePDAs for patients.

\section{Strengths and limitations}

This study has several strengths. First, multiple researchers were involved in the analysis of the interviews, which has strengthened our analysis because it gave us contesting as well as supplementary views which we discussed to reach consensus for the analysis. ${ }^{32}$ Second, this study generated similar results from two different settings and across disciplines, adding to the transferability. We acknowledge some limitations as well. First, recruitment for the interviews was based on availability of clinicians who completed the SDM training, which may have omitted those that were not willing to participate in the SDM training. However, we explicitly invited clinicians who were not able to use the ePDA on a frequent basis, adding to a diverse data sample. Finally, we did not interview patients because we were interested in the experiences of medical specialists. However, this could be an interesting additional source of information to expand the results, since interviewing medical specialists about their experiences in SDM could lead to a restricted viewpoint because of self-reporting their experiences.

\section{Future research}

Further research could use a quantitative design to examine what effect different settings (type of doctor, type of disease, type of patient) have on the patient-clinician dialogue. In our study, different settings across different specialties generated similar results but because of our qualitative study design, we are not able to quantify the effect of different settings. Second, to deepen knowledge on patient-clinician interactions and mechanisms during encounters, discourse analysis of video-taped encounters could be a useful method to apply.

\section{CONCLUSIONS}

Our findings hold two important lessons for practice. First, the results suggest that using ePDAs fostered the patient-clinician dialogue. Clinicians experienced a more structured conversation because of the content of the tool, and using the ePDAs prompted patients to ask more questions and the clinicians to discuss more information. Second, our study across several disciplines in two countries identified that a customised use and flexible workflow might improve and sustain the use of ePDAs in the future. Therefore, implementation efforts should focus on the flexible feature of the ePDA to integrate it into the clinician's workflow.

\section{Author affiliations}

${ }^{1}$ Radboud Institute for Health Sciences, Department of IQ healthcare, Radboudumc, Nijmegen, The Netherlands

${ }^{2}$ Knowledge Institute of the Dutch Association of Medical Specialists, Utrecht, The Netherlands

${ }^{3}$ Department of Obstetrics and Gynecology, Radboudumc, Nijmegen, The Netherlands

${ }^{4}$ The Dartmouth Institute for Health Policy and Clinical Practice, Hanover, New Hampshire, USA

${ }^{5}$ Department of Obstetrics and Gynecology, Amsterdam UMC Locatie AMC, Amsterdam, The Netherlands

Twitter Dunja Dreesens @DunjaDHH and Glyn Elwyn @glynelwyn

Acknowledgements We are grateful for Dr Tina Foster's help to prepare our study at the gynaecology departments in the USA, and for Mrs Molly Castaldo's help in conducting the semi-structured interviews with clinicians. Furthermore, we thank all medical specialists in The Netherlands and in New England, USA, who participated in the interviews.

Contributors EAR: analysis of Dutch and Dartmouth data, interpretation of all data; main drafting of the report; final approval of version to be published; accountable for all aspects of the report. DD: acquisition of some Dutch data; critically revising the report; final approval of version to be published; accountable for all aspects of the report. KV: designing the work; analysis of Dutch data; interpretation of Dutch data; minor revisions of the report; final approval of version to be published; accountable for all aspects of the report. MJM: designing the work; some analysis of Dutch data, critically revising the report; final approval of version to be published; accountable for all aspects of the report. TG: acquisition of Dutch data; analysis of Dutch data; interpretation of Dutch data; minor revisions of the report; final approval of version to be published; accountable for all aspects of the report. JTW: analysis of Dartmouth data; minor revisions of the report; final approval of version to be published; accountable for all aspects of the report. MD: analysis of Dartmouth data; interpretation of Dartmouth data; minor revisions of the report; final approval of version to be published; accountable for all aspects of the report. JAK: designing the work; critically revising the report; final approval of version to be published; accountable for all aspects of the report. GE: designing the work; critically revising the report; final approval of version to be published; accountable for all aspects of the report. JWMA: guarantor; designing the work; supervising acquisition, supervising analysis and interpretation of all data; critically revising the report; final approval of version to be published; accountable for all aspects of the report.

Funding The authors have not declared a specific grant for this research from any funding agency in the public, commercial or not-for-profit sectors.

Competing interests DD reports grants from ZonMw, during the conduct of the study. GE reports other from EBSCO, during the conduct of the study.

Patient consent for publication Not applicable.

Ethics approval This study involves human participants and was approved by The Committee for the Protection of Human Subjects, Dartmouth, USA: CPHS \#28362; CM0 Arnhem-Nijmegen (the Netherlands), file 2015-2016. All participants gave informed consent before taking part in the study. Participants gave informed consent to participate in the study before taking part.

Provenance and peer review Not commissioned; externally peer reviewed.

Data availability statement Data are available upon reasonable request. Data are de-identified transcribed interviews, available from the IQ healthcare Department of the Radboudumc upon request.

Supplemental material This content has been supplied by the author(s). It has not been vetted by BMJ Publishing Group Limited (BMJ) and may not have been 
peer-reviewed. Any opinions or recommendations discussed are solely those of the author(s) and are not endorsed by BMJ. BMJ disclaims all liability and responsibility arising from any reliance placed on the content. Where the content includes any translated material, BMJ does not warrant the accuracy and reliability of the translations (including but not limited to local regulations, clinical guidelines, terminology, drug names and drug dosages), and is not responsible for any error and/or omissions arising from translation and adaptation or otherwise.

Open access This is an open access article distributed in accordance with the Creative Commons Attribution Non Commercial (CC BY-NC 4.0) license, which permits others to distribute, remix, adapt, build upon this work non-commercially, and license their derivative works on different terms, provided the original work is properly cited, appropriate credit is given, any changes made indicated, and the use is non-commercial. See: http://creativecommons.org/licenses/by-nc/4.0/.

\section{ORCID iDs}

Ester A. Rake http://orcid.org/0000-0003-2262-3818

Dunja Dreesens http://orcid.org/0000-0002-5039-3370

Glyn Elwyn http://orcid.org/0000-0002-0917-6286

\section{REFERENCES}

1 Toerien M, Shaw R, Reuber M. Initiating decision-making in neurology consultations: 'recommending' versus 'option-listing' and the implications for medical authority. Sociol Health IIIn 2013;35:873-90.

2 Mulley AG, Trimble C, Elwyn G. Stop the silent misdiagnosis: patients' preferences matter. BMJ 2012;345:e6572.

3 Charles C, Gafni A, Whelan T. Shared decision-making in the medical encounter: what does it mean? (Or it takes at least two to tango). Soc Sci Med 1997;44:681-92.

4 Stiggelbout AM, Pieterse AH, De Haes JCJM. Shared decision making: concepts, evidence, and practice. Patient Educ Couns 2015;98:1172-9.

5 Stacey D, Légaré $\mathrm{F}$, Lewis $\mathrm{K}$, et al. Decision aids for people facing health treatment or screening decisions. Cochrane Database Syst Rev 2017;4: :CD001431.

6 Coates D, Clerke T. Training interventions to Equip health care professionals with shared decision-making skills: a systematic scoping review. J Contin Educ Health Prof 2020;40:100-19.

7 Grande SW, Faber MJ, Durand M-A, et al. A classification model of patient engagement methods and assessment of their feasibility in real-world settings. Patient Educ Couns 2014;95:281-7.

8 Garvelink MM, Jillissen M, Knops A, et al. Implementation of the three good questions-A feasibility study in Dutch hospital departments. Health Expect 2019;22:1272-84.

9 Shepherd HL, Barratt A, Jones A, et al. Can consumers learn to ask three questions to improve shared decision making? A feasibility study of the ASK (AskShareKnow) Patient-Clinician Communication Model(囚) intervention in a primary health-care setting. Health Expect 2016;19:1160-8.

10 Shepherd HL, Barratt A, Trevena LJ, et al. Three questions that patients can ask to improve the quality of information physicians give about treatment options: a cross-over trial. Patient Educ Couns 2011;84:379-85.

11 Scalia P, Durand M-A, Berkowitz JL, et al. The impact and utility of encounter patient decision AIDS: systematic review, meta-analysis and narrative synthesis. Patient Educ Couns 2019;102:817-41.

12 Elwyn G, Pickles T, Edwards A, et al. Supporting shared decision making using an option grid for osteoarthritis of the knee in an interface musculoskeletal clinic: a stepped wedge trial. Patient Educ Couns 2016:99:571-7.

13 Fay M, Grande SW, Donnelly K, et al. Using option grids: steps toward shared decision-making for neonatal circumcision. Patient Educ Couns 2016;99:236-42.
14 Elwyn G, Lloyd A, Joseph-Williams N, et al. Option grids: shared decision making made easier. Patient Educ Couns 2013;90:207-12.

15 Hahlweg P, Witzel I, Müller V, et al. Adaptation and qualitative evaluation of encounter decision AIDS in breast cancer care. Arch Gynecol Obstet 2019;299:1141-9.

16 Joseph-Williams N, Williams D, Wood F, et al. A descriptive model of shared decision making derived from routine implementation in clinical practice ('Implement-SDM'): qualitative study. Patient Educ Couns 2019;102:1774-85.

17 Dobler CC, Sanchez M, Gionfriddo MR, et al. Impact of decision AIDS used during clinical encounters on clinician outcomes and consultation length: a systematic review. BMJ Qual Saf 2019;28:499-510.

18 van der Weijden T, Post $\mathrm{H}$, Brand PLP, et al. Shared decision making, a buzz-word in the Netherlands, the pace quickens towards nationwide implementation.... Z Evid Fortbild Qual Gesundhwes 2017;123-124:69-74.

19 Braun V, Clarke V. Using thematic analysis in psychology. Qual Res Psychol 2006;3:77-101.

20 Tong A, Sainsbury P, Craig J. Consolidated criteria for reporting qualitative research (COREQ): a 32-item checklist for interviews and focus groups. Int J Qual Health Care 2007;19:349-57.

21 Aarts JWMet al. Training physicians to use an encounter decision aid in routine gynecology: impact on knowledge, attitudes and intentions. European Journal for Person Centered Healthcare 2019;7:70-4.

22 Alam S, Elwyn G, Percac-Lima S, et al. Assessing the acceptability and feasibility of encounter decision AIDS for early stage breast cancer targeted at underserved patients. BMC Med Inform Decis Mak 2016;16:147.

23 Elwyn G, Frosch DL, Kobrin S. Implementing shared decisionmaking: consider all the consequences. Implement Sci 2016;11:114.

24 Joseph-Williams N, Abhyankar P, Boland L, et al. What works in implementing patient decision AIDS in routine clinical settings? $A$ rapid realist review and update from the International patient decision aid standards collaboration. Med Decis Making 2021;41:272989x20978208:907-37.

25 Tong WT, Ng CJ, Lee YK, et al. What will make patients use a patient decision aid? A qualitative study on patients' perspectives on implementation barriers and facilitators. J Eval Clin Pract 2020;26:755-64.

26 Elwyn G, Durand MA, Song J, et al. A three-talk model for shared decision making: multistage consultation process. BMJ 2017;359:j4891.

27 Elwyn G, Rasmussen J, Kinsey K, et al. On a learning curve for shared decision making: interviews with clinicians using the knee osteoarthritis option grid. J Eval Clin Pract 2018;24:56-64.

28 Durand M-A, Alam S, Grande SW, et al. 'Much clearer with pictures': using community-based participatory research to design and test a picture option grid for underserved patients with breast cancer. BMJ Open 2016;6:e010008.

29 Schubbe D, Scalia P, Yen RW, et al. Using pictures to convey health information: a systematic review and meta-analysis of the effects on patient and consumer health behaviors and outcomes. Patient Educ Couns 2020;103:1935-60.

30 Rekenkamer A. Aanpak van laaggeletterdheid. Algemene Rekenkamer, 2016.

31 Goodman Met al. Literacy, Numeracy, and problem solving in Technology-Rich environments among US adults: results from the program for the International assessment of adult competencies 2012. First look. NCES 2014-008. National Center for Education Statistics, 2013.

32 Malterud K. Qualitative research: standards, challenges, and guidelines. Lancet 2001;358:483-8. 